\title{
Effect of Linked Rules on Business Process Model Understanding
}

\author{
Wang, Wei; Indulska, Marta; Sadiq, Shazia; Weber, Barbara
}

Published in:

Business Process Management

Link to article, DOI:

10.1007/978-3-319-65000-5_12

Publication date:

2017

Document Version

Peer reviewed version

Link back to DTU Orbit

Citation (APA):

Wang, W., Indulska, M., Sadiq, S., \& Weber, B. (2017). Effect of Linked Rules on Business Process Model Understanding. In Business Process Management (pp. 200-215). Springer. Lecture Notes in Computer Science Vol. 10445 https://doi.org/10.1007/978-3-319-65000-5 12

\section{General rights}

Copyright and moral rights for the publications made accessible in the public portal are retained by the authors and/or other copyright owners and it is a condition of accessing publications that users recognise and abide by the legal requirements associated with these rights.

- Users may download and print one copy of any publication from the public portal for the purpose of private study or research.

- You may not further distribute the material or use it for any profit-making activity or commercial gain

- You may freely distribute the URL identifying the publication in the public portal 


\title{
Effect of Linked Rules on Business Process Model Understanding
}

\author{
Wei Wang ${ }^{1}$, Marta Indulska ${ }^{2}$, Shazia Sadiq ${ }^{1}$, Barbara Weber ${ }^{3}$ \\ ${ }^{1}$ School of Information Technology and Electrical Engineering, \\ The University of Queensland, Brisbane, Australia \\ w.wang9@uq.edu.au, shazia@itee.uq.edu.au \\ ${ }^{2}$ University of Queensland Business School, \\ The University of Queensland, Brisbane, Australia \\ m. indulska@business.uq.edu. au \\ ${ }^{3}$ Department of Applied Mathematics and Computer Science, \\ Technical University of Denmark \\ bweb@dtu.dk
}

\begin{abstract}
.
Business process models are widely used in organizations by information systems analysts to represent complex business requirements and by business users to understand business operations and constraints. This understanding is extracted from graphical process models as well as business rules. Prior research advocated integrating business rules into business process models to improve the effectiveness of important organizational activities, such as developing shared understanding, effective communication, and process improvement. However, whether such integrated modeling can improve the understanding of business processes has not been empirically evaluated. In this paper, we report on an experiment that investigates the effect of linked rules, a specific rule integration approach, on business process model understanding. Our results indicate that linked rules are associated with better time efficiency in interpreting business operations, less mental effort, and partially associated with improved accuracy of understanding.
\end{abstract}

Keywords: Business Process Modeling, Business Rule Modeling, Cognitive Research

\section{Introduction}

In the Business Process Management (BPM) life cycle, the success of business process (re)design, analysis, and simulation are all underpinned by the assumption that the business activities are well understood. This understanding is extracted from graphical process models, which mainly focus on the temporal or logical relationships between business activities, as well as business rules, which are constraints and mandates that control the behavior of the process and business activities. Lack of good understanding of a business process and business rules that constrain the process can 
give rise to many risks. Users may inadvertently breach required standards of operation or make ill-informed decisions. Different stakeholders, such as process designers, information systems developers, and process participants may have inconsistent, or even conflicting, understanding of the same process. Ultimately, such inconsistencies hinder the effectiveness of important organizational activities and introduce risks of noncompliant process execution.

While all graphical process models generally integrate some aspects of rules (e.g. through control flow of the process), business rules can be represented in an integrated manner or in a separated manner. When represented in an integrated manner, they are shown graphically in a process model, either as textual annotations [1], as graphical links to external rules [2], or diagrammatically using the native notation of the graphical model [3], e.g. through a combination of sequence flows, activities and gateways. When modeled in a separated manner, rules are captured in separate documents or rule engines, and the relationships between the business process models and the rules are not explicitly represented in the process models. Traditionally, due to limited support for representation of business rules in graphical process modeling techniques [4], organizations often store such representations in separate text documents, spreadsheets, or disconnected business rule repositories [5]. Over the past two decades, prior work has argued for the need to model business rules in an integrated manner with business processes $[6,7]$, and a variety of integration methods $[1-3,6$, 8-11] and initial guidelines on rule integration [5] have been developed.

Arguments for such integration are typically based on an assumption of process improvement and shared understanding [5]. However, despite such arguments, and despite the different integration methods developed, if and to what extent such integration improves user understanding of the process models has not been investigated. In particular, while researchers have argued that integrated modeling can improve the understanding of business processes [5], this proposition has not been empirically evaluated. In this paper, we first present the theoretical foundation of the effects of rule integration on the cognitive activities of process model comprehension. With a focus on linked rules, a type of rule integration with process models, we then hypothesize the relationships between linked rules and process model understanding and report the results of our experiment to determine if linked rules can improve the understanding of process models.

\section{Background and Related Work}

A business process is a structured collection of activities that accomplishes a specific goal [12]. Such structures also involve business rules, which specify obligations, permissions, and restrictions that will limit the choice of approaches toward achieving a given goal [13]. Business rules typically can be classified as structural business rules (rules that describe constraints among data elements) and behavioral business rules (rules that describe the governing principles of process execution) [14]. Structural business rules are usually represented in data models, while behavioral business 
rules affect activities in process models. In this experiment, we focus on behavioral business rules.

Business process modeling and business rule modeling both focus on creating a representation of the organization's current and future practices. They are complementary approaches as they address distinct aspects of organizational practices. The overlap between business process models and business rules indicates a need to model the two related aspects together. Researchers argue that the integration of business rules into business process models can achieve better process model understanding [15-17], and improved governance, risk management and control [1, 18]. At the same time, however, researchers have identified a general lack of capability among process modeling languages to adequately represent business rules [4, 19, 20].

To solve this problem, a variety of integration methods and techniques have been developed since the publication of the first paper suggesting that business process and rule modeling approaches should be merged [21]. To name a few, McBrien et al. defined the structure of rules to couple business process models and rules [22]. Knolmayer et al. refined process modeling and linked the resulting models to workflow execution through layers of so-called Reaction Business Rules [23]. Kovacic et al. developed a meta-model to demonstrate how rules can link process, activity, events, data objects, and software components [13]. To summarize, three forms of integration of business process models and rules have been developed in literature viz. link integration, text integration, and diagrammatic integration. These approaches are summarized below and illustrated in Fig. 1.

Link integration. Link integration approaches incorporate information about the location of a related, externally documented, rule in a process model. Links can be static or automatic. In static link integration, the location information can be the section number and id, or the page number of the rule in a rulebook, thus allowing process users to locate the rule. Automatic link integration means the location information can be implemented as links, which will automatically navigate to the rule in the rule repository when the link is clicked. Notable contributions on link Integration are $[2,9]$.

Text integration. Text integration approaches represent the content of a rule textually in a business process model. For example, BPMN has a text annotation construct which allows users to put business rules into such an annotation construct in sentential format. Notable contributions on text integration are [1, 11].

Diagrammatic integration. While rules in link integration and text integration are represented in a sentential format, diagrammatic integration approaches represent rules in a diagrammatic format in a process model, using process modeling constructs such as sequence flows and gateways. A notable contribution on diagrammatic integration is [3]. 


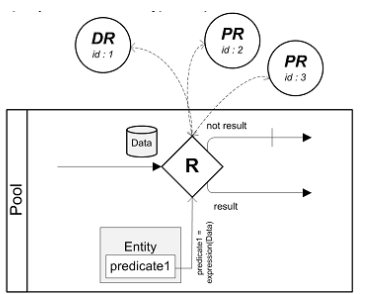

Link integration

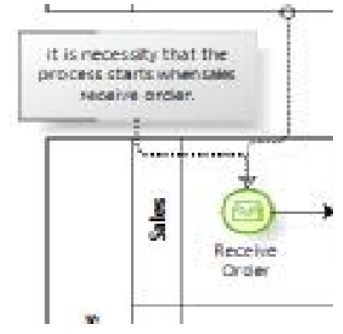

Text integration

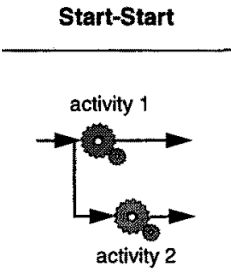

Diagrammatic integration

Fig. 1. Integration methods illustration

According to [24], the fundamental purpose of conceptual models is to improve users' understanding of the static and dynamic phenomena in a domain, and then to help developers and users to communicate and to serve as a basis for design. Conceptual model understanding has been widely studied in the past. To name a few, Wand et al. [25] proposed the Good Decomposition Model (GDM) that include minimality, determinism, losslessness, weak coupling, and strong cohesion as criteria for the design of conceptual models. Burton-Jones et al. [24, 26] studied the effect of decomposition of UML models and the combination effects of model decomposition quality and multiple forms of information on model understanding. Various factors affecting the understanding of process models, a typical type of conceptual models, such as the complexity, modularity and hierarchy of process models, individual cognitive ability, learning style, practice and education, have been studied (for an overview see [27]).

The argument that rule integration can improve process model understanding is the foundation that has motivated the development of different integration methods and techniques. The evaluation of the argument is critical to progress this research field. However, despite a considerable number of integration methods have been introduced using existing process modeling constructs, and despite many factors that can effect process model understanding have been identified, the question of whether integrating business rules into process models can improve the understanding of process models has not been theoretically analyzed nor empirically evaluated.

\section{Theoretical Background}

In this paper our aim is to study the link of integrating rules and process model understanding.

The limitations of diagrammatic integration are widely known due to the expressibility limitations of process modeling languages [1]. Similarly the drawbacks of rule integration through text annotations are duplicate and potentially inconsistent rule representations [28]. Hence in this paper we focus on a specific form of rule integration, namely link integration - an approach that points the model to the relevant rule, rather than duplicating that rule in the process model in either text or graphical form. 
Link integration approaches incorporate visual links that connect the relevant rules to a section of the model - i.e. the links are explicitly represented on the activities or gateways that the rules constrain. This approach thus makes the connections of rules and corresponding activities explicit, presumably reducing cognitive load required to mentally connect rules to the appropriate part of the process model [17]. When rules are modeled in a separated manner, on the other hand, they have to be semantically interpreted and manually matched by the model user to the relevant parts of the model. This is an error-prone process that requires the user to interpret the business rule against the background of the entire model to determine best fit. Accordingly, our first aim is to investigate the effect of link integration on process understanding accuracy, which means how well a process model is understood:

Hypothesis 1: Process models with linked rules are associated with better understanding accuracy compared with separated rules.

When rules are separated, all rules are organized as one set of rules, represented in some textual form (either plain text or in one of the business rule modeling languages). Finding the relevant rules that constrain a specific activity or gateway requires a comprehensive search and semantic interpretation of the set (e.g. linearly down the entire list of rules), which takes more time to mentally connect rules and a process model.

Accordingly, our second aim is to investigate the effect of rule linking on process understanding efficiency, focusing on how much time it takes a participant to review the process model and related rules to demonstrate understanding accuracy.

Hypothesis 2: Process models with linked rules are associated with better understanding time efficiency compared with separated rules.

As extra cognitive activities such as search and semantic interpretation are needed with rule linking, our third aim is to investigate the mental effort:

Hypothesis 3: Process models with linked rules are associated with less mental effort needed for understanding.

Despite the benefits, link integration is not without limitations. First, people using linked rules may focus on the interactions of specific rules and process components, without a holistic understanding of the process model and rules as a prerequisite, thus may have inaccurate understanding. Second, it can cause the attention switching effect [29], which means that users need to split their attention among multiple sources of information and mentally integrate them. Given separated rules as a whole list, one can choose to learn and assimilate more rules before switching attention to a process model, thus to reduce attention switches and time needed. It is therefore not clear to which degree the additional cognitive cost in terms of attention switching counterbalances the improvement in understanding. Thus, a study is needed to investigate this effect of business process and rule integration. To this end, we propose an experimental approach to test our hypotheses. 


\section{$4 \quad$ Research Method}

This study applies an experiment research method to explore differences between linked and separated business process models and rules. In this section we introduce our experimental design and describe our instruments, experiment settings and participants.

\section{Experiment Design}

The experiment is a single factor experiment. In our experiment, the use of linked rules is the considered factor, with factor levels "present" and "absent". We used two groups, two factor levels, and two domains in our experiment. Each group was tested with two domains separately, and for each domain, the two groups had different factor levels.

We have three main considerations in our between-subject design. First, our experiment environment only allows us to have one participant to do the experiment at a time. Second, the understanding performance depends on an individual's cognitive competence and experience. Thus, group imbalance is a challenge for betweensubject design. Third, we want to increase the generalization ability of the experiment in terms of domains, while controlling the learning effect.

Under these considerations, we designed our experiment as a balanced single factor experiment with repeated measurement, based on an experiment design from [30] which can increase the power of the experiment given the same number of participants [31]. The overall design is illustrated in Fig. 2. In this design, each participant will be tested for all factor levels and all domains, thus 1) more data will be collected than in a single run experiment, 2) two domains are tested to increase the generalizability of the results. The order of factor levels is reversed between groups, so the factor of order of treatment and learning effect are counterbalanced across groups. Please note that the forms of rule representation are inversed in the two runs. In the first run, group 1 are given linked rules and group 2 are given separated rules, while, in the second run, group 1 are given separated rules and group 2 are given linked rules.

Model 1

Model 2

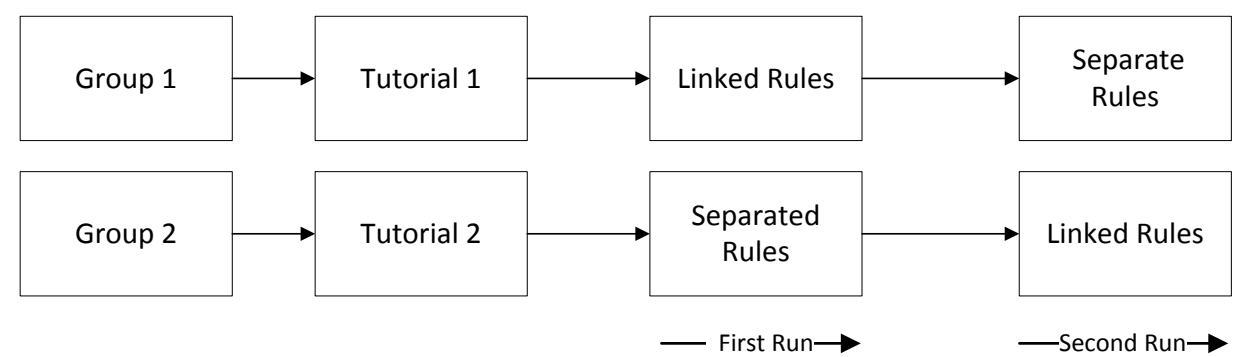

Fig. 2. Overall Experiment Approach 
As illustrated in Fig. 3, when linked rules are present, link buttons (labeled with "R") will be shown on activities and gateways in a process model, when a link button is clicked, the rules that are connected to the activity/gateway via the link button will be displayed on the "Relevant Rules" area on the right of the screen. When linked rules are absent, no link buttons will be shown in a process model, and all rules will be displayed in the "Relevant Rules" area on the right side of the screen.

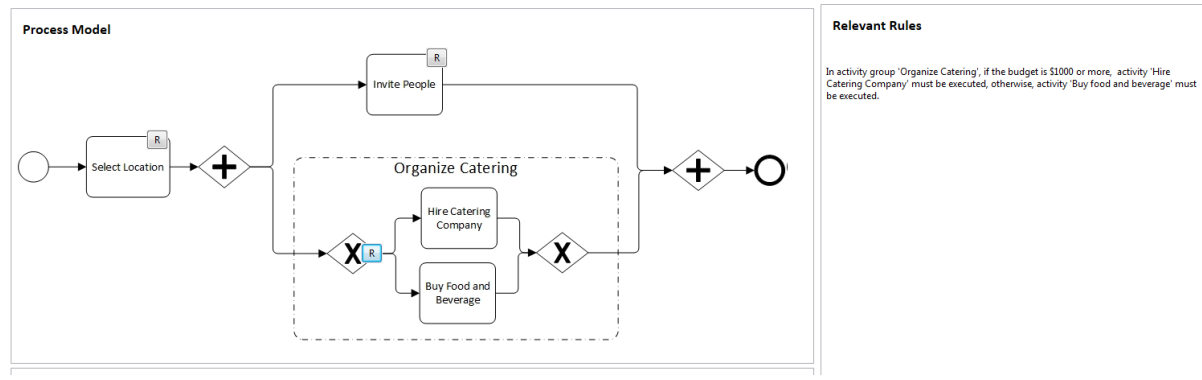

(a) Linked rules

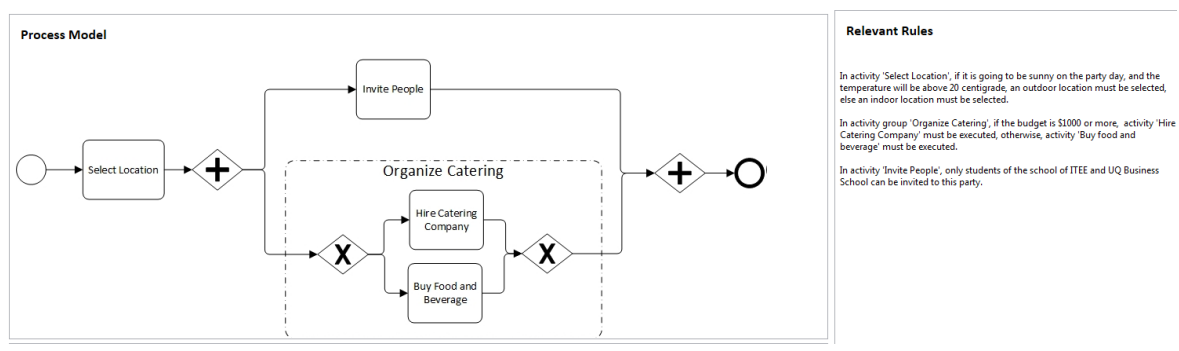

(b) Separated rules

Fig. 3. Independent variable illustration

\section{Measurements}

To measure the accuracy of understanding we use the percentage of correct answers to comprehension questions. We use the time from the point that a process model is displayed on the screen, to the point that the last question for this process model is answered as the measurement of time efficiency. To measure mental effort we use both an objective measure and a perception measure. We used the eye-fixation duration for each model as the objective measure. Eye-fixation is the maintaining of the visual gaze on a single location. Vision is suppressed during the eye saccade, and new information is acquired only during the fixation. Eye-fixation duration was proved to surpass pupil size as a mental effort measure [32]. As measure of perception of required mental effort, we asked each participant to select the model they perceived more difficult. 


\section{Instruments}

We briefly describe each part of the experimental instruments below.

Questionnaire. We have a pre-experiment questionnaire and a post-experiment questionnaire. The pre-experiment questionnaire asked participants if they are familiar with business process models and business rules, and if they are familiar with the knowledge domains of the process models in the experiment. The post-experiment questionnaire asked participants which model consumed most of their mental effort, their major, and which year they are in.

Tutorial and examples. The tutorial covered all BPMN elements and business rule concepts that participants would need to know to perform the tasks, e.g. activity, sequence, activity group, parallel gateway, exclusive gateway, and business rules. Example process models, rules, as well as questions and answers were provided after the tutorial. The instructions direct participants to study the process models, click the rule links, read the rules, and answer the questions. The order of treatments in the tutorial and examples are consistent with the order in the experiment.

Treatment design. To limit the learning effect, only 2 process models were used, and only 3 questions were asked for each model. The information needed from a process model and rules to answer a question are independent from each other thus the information learned from a previous question has little contribution to the current question. We designed process model A based on previous experiments [33, 34], and designed process model B to keep the complexity of the two models as close as possible. The rules and questions of the two process models are designed with the same cognitive load level in mind. The rules covered common rule violations such as time constraints, route selections, and data logic. To assimilate what happens in practice, several rules can control a single activity, and a violation of any of the rules will lead to a breach. We kept a variety of metrics of the two sets of models, rules, and questions the same or as close as possible to balance the experiment ${ }^{1}$.

\section{Settings}

The pre-experiment and post-experiment questionnaires were implemented in Qualtrics ${ }^{2}$. The tutorial and experiment were implemented as an Eclipse RCP application $^{3}$. The texts and diagrams were proved to be clearly visible from a distance of over $60 \mathrm{~cm}$ in the pilot test. As shown in Fig. 4, the screen was divided into three Areas, viz. Process Model Area, Relevant Rules Area and Questions Area. The complete process model and all the rules are displayed without the need of scrolling. No zooming is allowed in the application. All text and diagrams are in black and white so color blindness will not introduce bias to the experiment. We used Tobii Pro TX300, an eye

\footnotetext{
${ }^{1}$ The experiment can be downloaded from https://www.dropbox.com/s/g6jpb767m474vv2/experiment.rar?dl=0

${ }^{2}$ Qualtrics is a web-based survey platform. See: www.qualtrics.com.

${ }^{3}$ Eclipse RCP is a platform for building applications. See: https://wiki.eclipse.org/Rich_Client_Platform.
} 
tracker with a 23 -inch screen of a resolution of $1920 \times 1080$ that captures gaze data at $300 \mathrm{~Hz}^{4}$. The experiment was set in a lab. The lab has no window and the rooftop lights are the only light source. The materials, eye-tracker, and lights had the same settings for all participants.

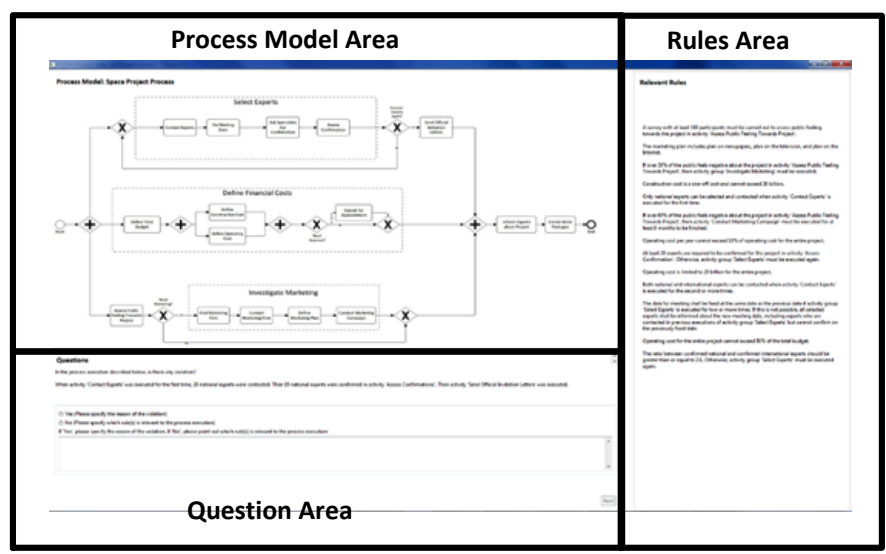

Fig. 4. Instrument Illustration

\section{Participants}

Students at an Australian university participated in this experiment voluntarily. Eight $\mathrm{PhD}$ students participated in the pilot tests. Fifty coursework students of an information systems course participated in the main experiment and were randomly assigned to two groups. Our sample size is considerable compared with other comparable experiments, which have sample sizes between 20-30 [32, 35]. All participants were required to have basic knowledge of flowcharts, UML or ER diagrams. We only used the most basic BPMN symbols and easily understandable daily English in the material which didn't require substantial experience from our participants. As an incentive, each student was offered a $\$ 30$ voucher for participation.

\section{$5 \quad$ Results}

For each dependent variable, we first checked if the dependent variable could be assumed to be normally distributed, following [30], we considered a variable to be normally distributed if the standardized skewness and standardized kurtosis were within the range of $[-2,2]$. If data of both groups were normally distributed, we checked whether the data met the assumption of equal variance using dependent Levene's test ${ }^{5}$ at the significance level of 0.05 , and then used the independent-sample

\footnotetext{
${ }^{4}$ For more specifications please see http://www.tobiipro.com/product-listing/tobii-pro-tx300.

${ }^{5}$ Levene's test is an inferential statistic used to assess the equality of variances for a variable calculated for two or more groups.
} 
$t$ test. If data in any group were not normally distributed, we used the Mann-Whitney $\mathrm{U}$ test ${ }^{6}$ across groups. We describe the results for each hypothesis in turn.

For Hypothesis 1, the correctness of question answers was normally distributed, and the data met the assumption of equal variance ( $p$ value of Levene's test is 0.61 for Model 1 and 0.25 for Model 2). We then ran independent-sample $t$ tests between group 1 and group 2, with the correctness of answers as the dependent variable, for the two models separately.

Table 1. Test of Hypotheses 1 - understanding accuracy

\begin{tabular}{|l|l|l|l|l|l|l|}
\hline & Group & $\mathrm{N}$ & Mean & Std. Dev & $\mathrm{t}$ & $\mathrm{p}$ (1-tailed) \\
\hline \multirow{3}{*}{ Correctness in Model 1 } & G1 & 25 & .73 & .25 & 1.37 & 0.088 \\
\cline { 2 - 8 } & G2 & 25 & .63 & .29 & & \\
\hline \multirow{2}{*}{ Correctness in Model 2 } & G1 & 25 & .63 & .36 & -1.77 & 0.042 \\
\cline { 2 - 8 } & G2 & 25 & .79 & .27 & & \\
\hline
\end{tabular}

Table 1 shows the results, which show that understanding accuracy was significantly correlated with the form of rule presentation in Model 2, but not in Model 1, which partially supports Hypothesis 1 .

Conclusion 1: Linked rules are partially associated with an improved understanding accuracy.

For Hypothesis 2, the time spent of group 2 in Model 2 was not normally distributed. We ran independent-sample Mann-Whitney tests between group 1 and group 2, with the time (from beginning to the end of answering the last question in each run) as the dependent variable. The test result of Hypothesis 2 is shown in Table 2. Table 2 shows that time used in each model is related to the form of rule presentation, supporting Hypothesis 2 at a significance level of 0.05 .

Table 2. Test of Hypothesis 2: understanding efficiency

\begin{tabular}{|l|l|l|l|l|l|}
\hline & Group & N & Mean & $\begin{array}{l}\text { Std. Devia- } \\
\text { tion }\end{array}$ & p (1-tailed) \\
\hline \multirow{2}{*}{ Time used in Model 1 } & G1 & 23 & 368.76 & 110.23 & \multirow{2}{*}{0.015} \\
\cline { 2 - 6 } & G2 & 25 & 481.18 & 218.10 & \multirow{2}{*}{0.009} \\
\hline \multirow{2}{*}{ Time used in Model 2 } & G1 & 23 & 468.57 & 173.06 & \\
\cline { 2 - 5 } & G2 & 25 & 370.46 & 116.88 & \\
\hline
\end{tabular}

Conclusion 2: Linked rules are associated with increases in understanding efficiency.

${ }^{6}$ The Mann-Whitney U test is used to compare differences between two independent groups when the dependent variable is not normally distributed. 
For Hypothesis 3, the eye-fixation durations in the two runs were not normally distributed. We therefore ran independent-sample Mann-Whitney tests for the two runs separately. The objective test of Hypothesis 3 is shown in Table 3. From Table 3 we can see that the mental effort is associated with the type of rule presentation, supporting Hypothesis 3 at a significance level of 0.05 .

Table 3. Test of Hypothesis 3: objective mental effort

\begin{tabular}{|l|l|l|l|l|l|}
\hline & Group & N & Mean & Std. Deviation & p (1-tailed) \\
\hline \multirow{2}{*}{ Fixation duration in Model 1 } & G1 & 23 & 322.98 & 100.30 & \multirow{2}{*}{0.024} \\
\cline { 2 - 6 } & G2 & 25 & 411.43 & 188.22 & \\
\hline \multirow{2}{*}{ Fixation duration in Mode1 2 } & G1 & 23 & 409.68 & 159.94 & \multirow{2}{*}{0.007} \\
\cline { 2 - 5 } & G2 & 25 & 318.53 & 102.31 & \\
\hline
\end{tabular}

The results of the perception of mental effort are shown in Table 4. In group 1, 0 participants selected Model 1 (linked rules), while 23 participants selected Model 2 (separated rules) as the model requiring the more mental effort. Two participants selected 'equal' as the answer. In group 2, 11 participants selected Model 1 (separated rules), while 6 participants selected Model 2 (linked rules) as the model requiring more mental effort. Eight participants selected 'equal' as the answer. From Table 4 we can intuitively see that participants indicate that models with separated rules require more mental effort, regardless of model content (model 1 or model 2).

Table 4. Perception of mental effort

\begin{tabular}{|l|l|l|}
\hline & Group 1 & Group 2 \\
\hline Model 1 requires more mental effort & 0 (linked rules) & 11 (separated rules) \\
\hline Model 2 requires more mental effort & 23 (separated rules) & 6 (linked rules) \\
\hline Equal & 2 & 8 \\
\hline
\end{tabular}

To statistically compare linked and separated rules, we coded the perception answers as follows: When a model with linked rules was selected as the model that required more mental effort, linked rules were assigned 2 points. When the model with separated rules was selected as the model that required more mental effort, separated rules were assigned 2 points. When a participant selected the two models as equal, both linked rules and separated rules were assigned 1 point. We used a $t$ test for the difference in average mental effort perception between linked and separated rules. Table 5 shows that mental effort in linked rules is significantly smaller than in separated rules.

Table 5. Coded mental effort

\begin{tabular}{|l|l|l|l|l|}
\hline & $\mathrm{N}$ & Coded Mean & Std. Deviation & $\mathrm{p}$ (1-tailed) \\
\hline Linked rules & 50 & 0.44 & 0.70 & \multirow{2}{*}{0.000} \\
\cline { 1 - 4 } Separated rules & 50 & 1.56 & 0.70 & \\
\hline
\end{tabular}


Conclusion 3: Linked rules are associated with reduced mental effort required for model understanding.

\section{Discussion}

Our results support hypothesis 2 and hypothesis 3, indicating that linked rules are associated with increases in understanding efficiency and reduced mental effort required for model understanding. While hypothesis 1 has only partial support. For the results of hypothesis 1 , the $p$ value for Model 1 was greater than 0.05 , indicating a lack of statistical significance. To explore this result further, first we compared the two models, and the metrics comparison showed that the two sets of models, rules and questions are the same or close in all the metrics. Second, we investigated answer correctness and time spent of each model. The statistics showed that the two sets of models, rules, and questions had no significant difference (with $\mathrm{p}=0.647$ and $\mathrm{p}=$ 0.822 respectively). Thus, we concluded that there was no bias between Model 1 and Model 2. Finally, we broke down the correctness of answers to each question to explore the lack of statistical significance of the differences between linked and separated rules in Model 1. As shown in Fig. 5, the result of question 1 shows that the group with linked rules had lower understanding accuracy than the group with separated rules, which is against Hypothesis 1, while the correctness of all other 5 questions indicates the support of Hypothesis 1. We assume that one possible reason is that the participants had not learnt how to use linked rules well when they met the first question. Recall that we had to balance time with fatigue and tracking data accuracy and thus had a time constrain in the experiment, so we used a simple illustration of linked rules (See Fig. 3) in the training material, compared with the models and rules in the formal experiment what were much more complex and challenging. Thus, participants may not quickly find how to utilize rule links.

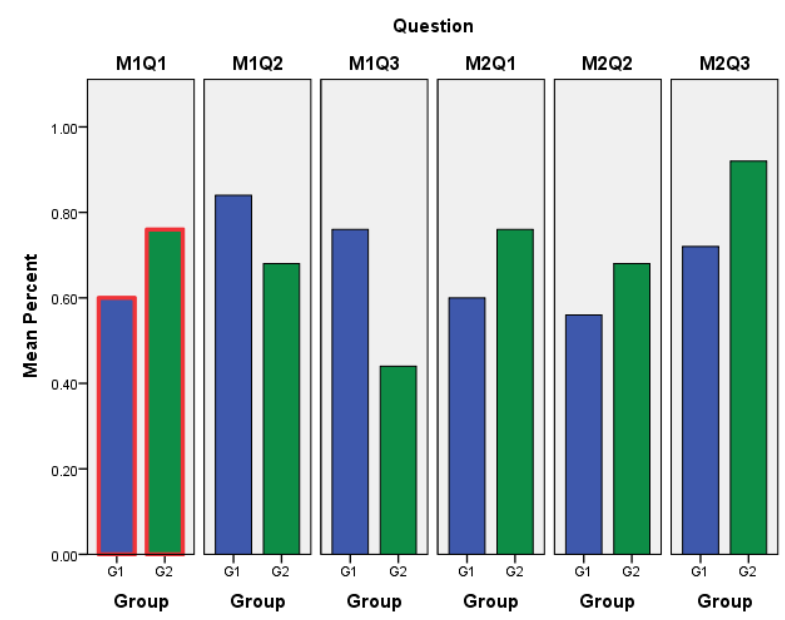

Fig. 5. Answer correctness breakdown to each question 
Our study is not without limitations. In terms of internal validity, the different layout of screen areas could possibly affect the results It is possible that the experiment results will be different if we change the location of each area. In terms of construct validity, we operationalized each construct in our study in limited ways. The questions were designed to test the understanding of the effect of business rules on business process models. Following [36], it would have been ideal if we had measured the perceived quality and efficiency of understanding, and asked questions only about a process model itself. Thus, our research results are limited to the treatments, measurements and questions that we used. Finally, in terms of external validity, we cannot say that the process models, rules, and questions we used faithfully reflect those used in organizations in practice. Organizations may use more complex process models and lager number of rules, and the tasks may be more challenging. The use of students as participants could also weaken the generalization ability of the results.

\section{$7 \quad$ Conclusions and Outlook}

In this paper, we have studied the relationship between rule integration and business process model understanding. Rules can be integrated into process models in a variety of ways, and in this paper, we report on our findings based on a specific form of rule integration, namely linked rules. We focused on 3 aspects of understanding: understanding accuracy, time efficiency, and mental effort. Our study results presented 3 conclusions: (1) The association between linked rules and understanding accuracy is partially supported. (2) Linked rules are significantly associated with improved time efficiency. (3) Linked rules are significantly associated with reduced mental effort. Our conclusions are drawn from an experiment design that utilized an eyetracker. The design of the experiment provides a methodological contribution towards the study of process model understanding. Opportunities exist for future research to perform similar experiments on different rule integration methods such as annotation and diagrammatical integration [37] and investigate the effects on process model understanding.

Business rules have a broad scope, and business rules can be quite varied in many aspects such as change frequency, complexity and governance responsibility [37]. Thus, the best way for each rule to be integrated into a process model can be different. The characteristics of business rules or different rule categories can influence which integration method has the best performance in terms of process model understanding. Quite a few business rule classification frameworks such as [38, 39] exist in literature. Finding the connection between type of rule and the best corresponding integration approach to improve process model understanding will be a valuable topic for future research. 


\section{References}

1. Cheng, R., Sadiq, S., Indulska, M.: Framework for Business Process and Rule Integration: A Case of BPMN and SBVR. In: Business Information Systems. pp. 13-24. Springer (2011).

2. Sapkota, B., van Sinderen, M.: Exploiting rules and processes for increasing flexibility in service composition. In: Enterprise Distributed Object Computing Conference Workshops (EDOCW), 2010 14th IEEE International. pp. 177-185. IEEE (2010).

3. Kappel, G., Rausch-Schott, S., Retschitzegger, W.: Coordination in workflow management systems - A rule-based approach. In: Conen, W. and Neumann, G. (eds.) Coordination Technology for Collaborative Applications. pp. 99-119. Springer Berlin Heidelberg (1998).

4. Recker, J., Rosemann, M., Green, P.F., Indulska, M.: Do ontological deficiencies in modeling grammars matter? MIS Quarterly. 35, 57-79 (2011).

5. Zur Muehlen, M., Indulska, M., Kittel, K.: Towards integrated modeling of business processes and business rules. In: Proceedings of the 19h Australasian Conference on Information Systems (ACIS)-Creating the Future: Transforming Research into Practice, Christchurch, New Zealand. pp. 690-697. Citeseer (2008).

6. Habich, D., Richly, S., Demuth, B., Gietl, F., Spilke, J., Lehner, W., Assmann, U.: Joining Business Rules and Business Processes. Proc. of IT. (2010).

7. Zur Muehlen, M., Indulska, M.: Modeling languages for business processes and business rules: A representational analysis. Information systems. 35, 379-390 (2010).

8. Kluza, K., Kaczor, K., Nalepa, G.J.: Enriching Business Processes with Rules Using the Oryx BPMN Editor. In: Rutkowski, L., Korytkowski, M., Scherer, R., Tadeusiewicz, R., Zadeh, L.A., and Zurada, J.M. (eds.) Artificial Intelligence and Soft Computing, Pt Ii. pp. 573-581 (2012).

9. Nalepa, G.J., Kluza, K., Kaczor, K.: Proposal of an Inference Engine Architecture for Business Rules and Processes. In: Rutkowski, L., Korytkowski, M., Scherer, R., Tadeusiewicz, R., Zadeh, L.A., and Zurada, J.M. (eds.) Artificial Intelligence and Soft Computing. pp. 453-464. Springer Berlin Heidelberg (2013).

10. Milanovic, M., Gasevic, D., Rocha, L.: Modeling Flexible Business Processes with Business Rule Patterns. In: Enterprise Distributed Object Computing Conference (EDOC), 2011 15th IEEE International. pp. 65-74 (2011).

11. Governatori, G., Shek, S.: Rule based business process compliance. Proceedings of the RuleML2012@ECAI Challenge.5 (2012).

12. Hammer, M., Champy, J.: Reengineering the corporation: A manifesto for business revolution. Business Horizons. 36, 90-91 (1993).

13. Kovacic, A., Groznik, A.: The business rule-transformation approach. In: 26th International Conference on Information Technology Interfaces, 2004. pp. 113117 Vol.1 (2004).

14. Nayak, N., Linehan, M., Nigam, A., Marston, D., Jeng, J.-J., Wu, F.Y., Boullery, D., White, L.F., Nandi, P., Sanz, J.L.C.: Core business architecture for a serviceoriented enterprise. IBM Systems Journal. 46, 723-742 (2007). 
15. Rabova, I.: Methodology of the Enterprise Architecture creating and the role of the Enterprise Architecture in rural development. Agricultural EconomicsZemedelska Ekonomika. 56, 334-340 (2010).

16. Skersys, T., Tutkute, L., Butleris, R., Butkiene, R.: Extending BPMN Business Process Model with SBVR Business Vocabulary and Rules. Information Technology And Control. 41, 356-367 (2012).

17. Wang, W., Indulska, M., Sadiq, S.: Cognitive Efforts in Using Integrated Models of Business Processes and Rules - Semantic Scholar. In: Proceedings of the 28th International Conference on Advanced Information Systems Engineering (CAiSE Workshop). Springer, Ljubljana, Slovenia (2016).

18. Ly, L.T., Rinderle-Ma, S., Göser, K., Dadam, P.: On enabling integrated process compliance with semantic constraints in process management systems. Information Systems Frontiers. 14, 195-219 (2012).

19. Green, P.F., Rosemann, M.: Perceived Ontological Weaknesses Of Process Modeling Techniques: Further Evidence. In: ECIS. pp. 312-321 (2002).

20. Herbst, H., Knolmayer, G., Myrach, T., Schlesinger, M.: The specification of business rules: A comparison of selected methodologies. In: Methods and associated tools for the information systems life cycle. pp. 29-46 (1994).

21. Krogstie, J., McBrien, P., Owens, R., Seltveit, A.H.: Information systems development using a combination of process and rule based approaches. In: Andersen, R., Jr, J.A.B., and Sølvberg, A. (eds.) Advanced Information Systems Engineering. pp. 319-335. Springer Berlin Heidelberg (1991).

22. McBrien, P., Seltveit, A.H.: Coupling process models and business rules. In: Information Systems Development for Decentralized Organizations. pp. 201217. Springer (1995).

23. Knolmayer, G., Endl, R., Pfahrer, M.: Modeling Processes and Workflows by Business Rules. In: Aalst, W. van der, Desel, J., and Oberweis, A. (eds.) Business Process Management. pp. 16-29. Springer Berlin Heidelberg (2000).

24. Burton-Jones, A., Meso, P.N.: Conceptualizing Systems for Understanding: An Empirical Test of Decomposition Principles in Object-Oriented Analysis. Information Systems Research. 17, 38-60 (2006).

25. Wand, Y., Weber, R.: An ontological model of an information system. IEEE transactions on software engineering. 16, 1282-1292 (1990).

26. Burton-Jones, A., Meso, P.N.: The Effects of Decomposition Quality and Multiple Forms of Information on Novices' Understanding of a Domain from a Conceptual Model. Journal of the Association for Information Systems. 9, 748-802 (2008).

27. Figl, K.: Comprehension of Procedural Visual Business Process Models-A Literature Review.

28. Loucopoulos, P., Kadir, W.M.N.W.: BROOD: business rules-driven object oriented design. Journal of Database Management (JDM). 19, 41-73 (2008).

29. Sweller, J., Chandler, P.: Why Some Material Is Difficult to Learn. Cognition and Instruction. 12, 185-233 (1994).

30. Reijers, H.A., Mendling, J., Dijkman, R.M.: Human and automatic modularizations of process models to enhance their comprehension. Information Systems. 36, 881-897 (2011). 
31. Charness, G., Gneezy, U., Kuhn, M.A.: Experimental methods: Between-subject and within-subject design. Journal of Economic Behavior \& Organization. 81, 18 (2012).

32. Meghanathan, R.N., van Leeuwen, C., Nikolaev, A.R.: Fixation duration surpasses pupil size as a measure of memory load in free viewing. Front Hum Neurosci. 8, (2015).

33. Zugal, S., Pinggera, J., Weber, B., Mendling, J., Reijers, H.A.: Assessing the Impact of Hierarchy on Model Understandability - A Cognitive Perspective. In: Kienzle, J. (ed.) Models in Software Engineering. pp. 123-133. Springer Berlin Heidelberg (2011).

34. Zugal, S.: Applying Cognitive Psychology for Improving the Creation, Understanding and Maintenance of Business Process Models.

35. Haji, F.A., Rojas, D., Childs, R., de Ribaupierre, S., Dubrowski, A.: Measuring cognitive load: performance, mental effort and simulation task complexity. Med Educ. 49, 815-827 (2015).

36. Wang, W., Indulska, M., Sadiq, S.: To Integrate or not to Integrate - The Business Rules Question. In: Proceedings of the 28th International Conference on Advanced Information Systems Engineering. Springer, Ljubljana, Slovenia (2016).

37. Zoet, M., Versendaal, J., Ravesteyn, P., Welke, R.J.: Alignment of business process management and business rules. In: Proceedings of the 19th European Conference on Information Systems. p. 34., Helsinki, Finland (2011).

38. Hashmi, M., Governatori, G., Wynn, M.T.: Normative requirements for business process compliance. (2013).

39. Campbell, D.T., Fiske, D.W.: Convergent and discriminant validation by the multitrait-multimethod matrix. Psychological Bulletin. 56, 81-105 (1959). 